\title{
Redesign of Functional Kaba (Blouse) Apparel for Ghanaian Breastfeeding Mothers
}

\section{Rowena Fatchu Kansanba1, Vincentia Okpattah' ${ }^{2}$, Mercy Ampofowah Osei ${ }^{3}$, Solomon Marfo Ayesu', Abraham Ekow Asmah ${ }^{2}$}

\author{
${ }^{1}$ Department of Fashion Design and Textile Studies, Kumasi Technical University (KsTU), Kumasi, Ghana \\ ${ }^{2}$ Department of Integrated Rural Art and Industry, Kwame Nkrumah University of Science and Technology (KNUST), Kumasi, \\ Ghana \\ ${ }^{3}$ Department of Fashion, Bluecrest University College, Accra, Ghana \\ Email: rfatchu12@gmail.com, ovishgh@yahoo.com,ampofowah63@gmail.com, solomaxxy@rocketmaill.com, \\ ekowasmah@yahoo.co.uk
}

How to cite this paper: Kansanba, R.F., Okpattah, V., Osei, M.A., Ayesu, S.M., and Asmah A.E. (2021) Redesign of Functional Kaba (Blouse) Apparel for Ghanaian Breastfeeding Mothers. Journal of Textile Science and Technology, 7, 41-54. https://doi.org/10.4236/jtst.2021.71004

Received: December 29, 2020

Accepted: February 17, 2021

Published: February 20, 2021

Copyright $\odot 2021$ by author(s) and Scientific Research Publishing Inc. This work is licensed under the Creative Commons Attribution International License (CC BY 4.0).

http://creativecommons.org/licenses/by/4.0/

\begin{abstract}
$K a b a$ is considered to be a kind of traditional apparel worn by the women in Ghana. Though, it's worn to accentuate female modesty, chastity and elegance among the women folks. The modern day woman faces a lot of physical and emotional challenges when it comes to breastfeeding. There is a need to redesign and improve upon the kaba apparel for breastfeeding purposes. This study, therefore, sought to redesign functional kaba apparel for breastfeeding mothers. Based on the apparel design framework of Lamb and Kallal, design requirements were identified from breastfeeding mothers and incorporated into the designed apparel. Five prototype designs were made and tested by three selected mothers towards effective breastfeeding. Findings revealed that the prototype apparels made of fasteners and openings in the horizontal, diagonal and vertical directions with zippers and buttons were said to be suitable, comfortable to use and hence acceptable for implementation. This added functional part of the apparel boosted the comfort and confidence of the mother without compromising on their expressive values.
\end{abstract}

\section{Keywords}

Kaba Ensemble, Redesign, Breastfeeding Mothers, Effective Breastfeeding

\section{Introduction}

Clothing has had great meaning over the years, indicating the aesthetic abilities of its users, artistic imaginations of garment makers and expression of cultural values. Clothing is a manufactured article which reflects details about the people 
who wear it and also tells the story about the wearer [1]. It is also a basic need of mankind throughout the world as it helps make impression on people [2].

Clothing plays an important role by protecting us from various external factors, provides physiological as well as psychological comfort to humans. Thus, our preferences and behaviour regarding clothing are influenced by factors such as physical (protection), psychological (adornment and identification) and socio-cultural (modesty/immodesty and status). Clothing is not merely motivated by these alone but also influenced by the environment [3]. Human behaviour takes place within a cultural context. In spite of these, the lactating woman faces various psychosocial barriers to breastfeeding, in addition to the day-to-day emotional and physical challenges in public places [4]. Yet, apart from natural occurrences like the weather that informs the wearer, accepted standard within an environmental context tends to guide our choice of clothing.

Unlike fashion clothing which is a product of the designer's creative instincts, designing functional clothing begins and ends with the user's specific requirements in mind [5]. The writer further postulates that these requirements whether for performance or comfort are determined by the environment and the activities he or she performs. She thus defines functional clothing is "all such types of clothing or assemblies that are specifically engineered to deliver a pre-defined performance or functionality to the user over and above its normal functions" [6]. Furthermore, such types of clothing are made from a mix of innovative materials and functionality which in this case would imply an added value or function that the garment is expected to perform. These clothing are made out of advanced or creative use of materials or features intended to add value to the garment in solving the problem of the user. These include: sportswear, dinner wear, those that aid in the easy movement in the physically challenged or for special necessities such as pregnancy and lactation. The development of these functional clothes helps to improve upon the quality of life of their intended user whose body shape, size and mobility have been affected as a result of their predicament [7]. In considering functional properties within an apparel design context, vital considerations are made to the manufacturing of the product in terms of its utility, protection, thermal comfort, fit and ease of movement as some examples of functional requirements a designer must seek [8]. Rosenbald-Walin [9] highlights the importance of considering the demand of such situation in developing apparel product. In light of the above, the kaba or apparel for lactation considered these options to go beyond its normal aesthetic and expressive appearance to improve upon the apparel to serve the intended need.

Understanding the human body becomes a critical component of the design process [10]. A key characteristic of User Product Development method is a "systematic analysis of the relation between user, product, task, and environment," [11]. This method can be used in functional and technical apparel research [12]. While determining user needs, it is necessary to define and measure deficiencies in products against the users' ideal of those product(s). This process is known as a needs assessment [13] [14]. Needs assessments have been success- 
fully used by apparel researchers when designing apparel for special user groups [15] [16] [17].

At the centre of Lamb and Kallal's model is the target consumer who happens to be the end user whom the designer directly targets the apparel to be made for. The consumer needs must be analysed in order for a designer to develop a profile of the user that can include demographics, psychographic information, physical characteristics, activities and preferences [8]. The intention is to clarify the consumer needs and wants in terms of the situation in which the apparel is used. Culture can be seen as a social phenomenon that identifies and associates an individual to a group of people who believe in one philosophy that reflects in all facets of their lives including clothing and fashion [18]. "What people know, feel, think, make and do" in a nut shell can be summarized as culture [19]. As people create structures for what they know, feel, think, make and do, meaning becomes attached to items such as clothing. These meanings are attributed to the social interactions an individual has with others [20].

Kaba in Ghana is a kind of fashion apparel that has come to stay among women since time immemorial. The apparel was and is worn to accentuate female modesty, chastity and elegance. Kaba is one of the important apparels which usually form an integral part of the wardrobe of the breastfeeding mother. This is partly because of the cover cloth which forms part of the local ensemble usually used to carry the baby at the back of the mother. The kaba ensemble which is made up of the kaba (blouse), slit (skirt) and cover cloth remains the most sought after apparel among women in Ghana. Dressmakers and designers alike are creating varieties of the apparel resulting in the promotion of dynamism and complexity of designs. This is evident in the proliferation of kaba magazines; calendar's and catalogues for various occasions. These attest to the ingenuity of kaba designers and the rapid pace at which the styles are adapted and redesigned without addressing its challenges.

The kaba ensemble therefore remains the symbol of motherhood and the most sought after apparel among Ghanaian women. For these reasons, the study sought to identify the clothing ( $k a b a$ ) and its related problems of the breastfeeding mother and to redesign $k a b a$ (blouse) that is technically and functionally suitable for breastfeeding mothers without compromising on its expressive values.

\section{Materials and Methods}

The study employed the qualitative research method and triangulated the data collection through obtrusive and unobtrusive observations. Participants were randomly selected and interviewed. Apparel production was based on the conceptual apparel design framework of Lamb and Kallal. The following notable fabrics were used; African print fabrics also known as "mummy cloths" in Ghana and beyond are made up of wax, java and fancy with conceptualized designs which are symbolic and significant to the Ghanaian African culture [21]. African prints have philosophical significance. These prints have names that could easily 
depict or explain the beliefs, practices and culture of Ghanaians. The patterns in the prints tell stories of importance to the wearer, such as proverbs, poems and traditional African tales. The colours also hold philosophical significance as they can represent social standing, age, tribal orientation and marital status [21]. Polyester was used as an underlining to add weight to the apparel and drape. Zippers and buttons were used as closure for the openings while lace, bias and plain cotton fabric were used in embellishing the garment.

The study was carried out from three post-natal units within the Kumasi metropolis of the Ashanti Region of Ghana. One hundred and eigthy (180) breastfeeding mothers were interviewed. The functional garments redesigned were given to three mothers representing each of the postnatal hospitals to assess their comfortability, suitability and acceptability towards effective breastfeeding. The conceptual framework employed for the design is illustrated below (Figure 1).

\subsection{Problem Identification}

The Ghanaian kaba is one of the three types of female apparels worn in the Ashanti region of Ghana [22]. These are the kaba ensemble, dansinkran ensemble and dresses. The three piece kaba ensemble sewn out of a half piece of cloth thus six yards, is made up of the kaba (blouse), slit (skirt) and the extra two yard cloth usually used as a stole, headgear, wrapped over the slit or used to carry babies at the back of the mother. The choice of the kaba ensemble in this case, was found to be associated with Ghanaian women as a symbol of modesty, chastity and the fact of being Ghanaian [23]. It is one the culturally accepted apparels in Ghana hence cannot be ignored by mothers. All these notwithstanding, the Ghanaian kaba ensemble is challenged when it comes to breastfeeding. The once all important apparel seems to be relegated to the background when it comes to

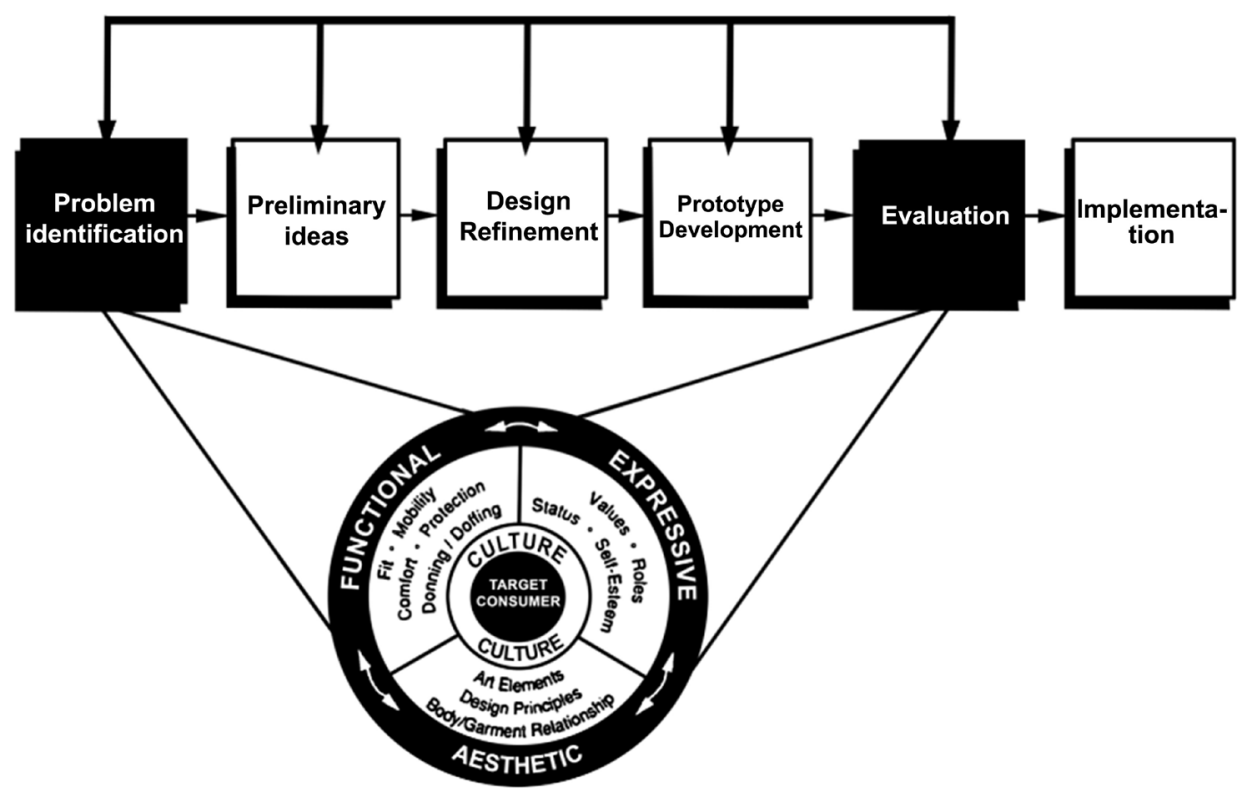

Figure 1. Apparel design framework [8]. 
breastfeeding among mothers. Culture should be what influences people to consider what is acceptable when solving various design problems [8]. The kaba (blouse) most of the time has its opening at the back while other embellishment are made at the front. The general fitting nature of the kaba and most clothing makes the mother uncomfortable when breastfeeding because, the breastfeeding mother has to unzip if the fastener is at the back, raise the blouse or pull down the neckline of the blouse. These inconveniences of the kaba blouse and other dresses or blouses were observed to expose some other parts of the body such as shoulders, back, chest and or tummy (Figures 2-4) making some mothers uncomfortable breastfeeding in public.

These relates to breastfeeding mothers asked to leave public places because exposing their breast to nurse might be offensive to others [2]. Most breastfeeding wears have limited longevity as the garments are used for a maximum of six months and rendered non-serviceable when the baby stops feeding [24]. The

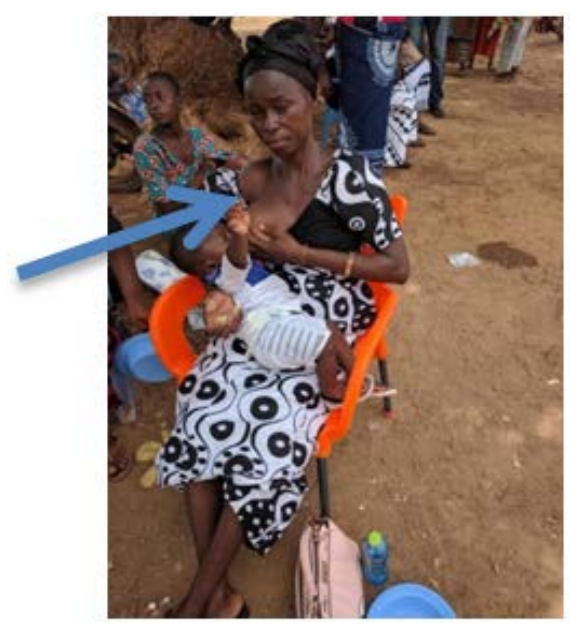

Figure 2. Mother exposing her shoulder (Field survey).

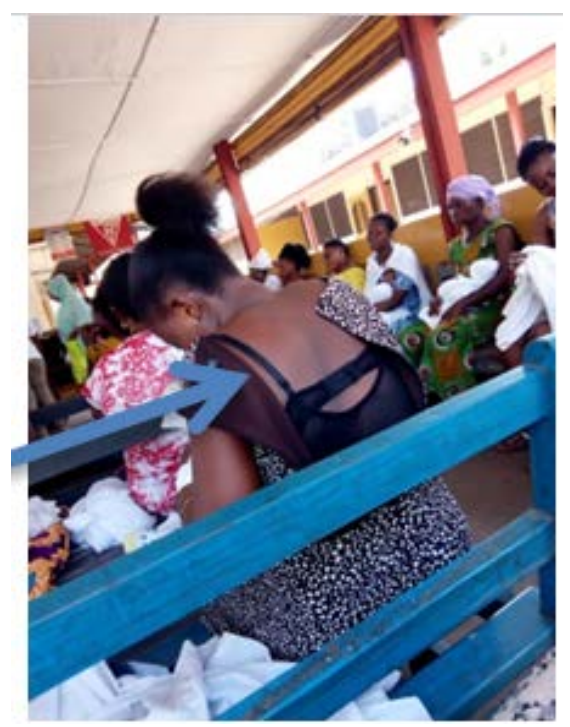

Figure 3. Mother exposing her back (Field survey). 


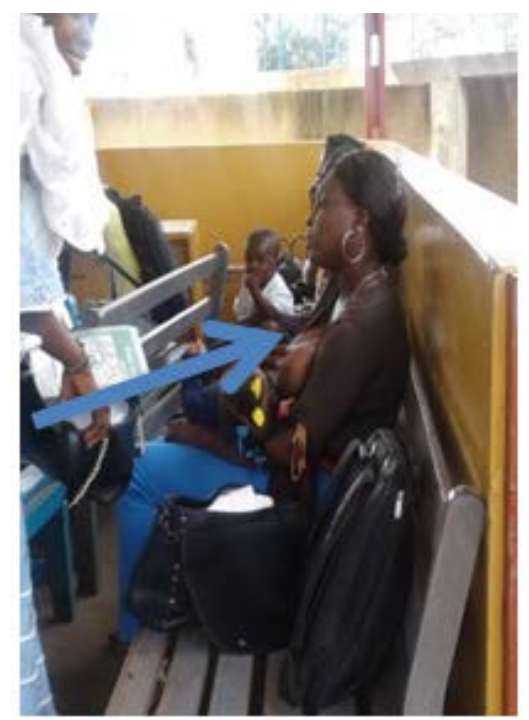

Figure 4. Mother exposing her chest (Field survey).

need to redesign the kaba (blouse) apparel and make it user friendly for use before, during and after breastfeeding was to maintain the culture of modesty among young mothers so as not to relegate the local ensemble to the background. An interviewee who happened to be a secretary and a mother of five said though she loved to wear the apparel, the uncomfortability of wearing them and breastfeeding led her to abandon them whenever she had a baby. Another mother also complained of being humiliated in public transport because she could not breastfeed her infant as a result of the kaba style she wore. The graph (Figure 5) shows the comfortability level of the breastfeeding mothers with regards to their kaba apparel and breastfeeding.

From the bar chart above (Figure 5), 92 respondents representing 51\% confirmed they felt uncomfortable breastfeeding in their kaba, while 52 respondents representing $29 \%$ said they have been managing the kaba though they felt uncomfortable breastfeeding in them. These responses have left most of them putting aside the apparel so as to enable them breastfeed in other forms of clothing. These findings refute [23] that, young ladies would save money just to acquire a number of African prints to sew the kaba ensemble after marriage and particularly after childbirth. The remaining 36 respondents representing $20 \%$ said they were satisfied with their kind of kaba style and breastfeeding possibly because of their low cut necklines which confirm [25] that necklines are important features of breastfeeding apparel in tops, dresses, tanks and bras. Scoop, $\mathrm{V}$-neck and cross-over necklines are typical because they allow easy access for breastfeeding by simply pulling the garment down or up depending on the apparel feature. These certainly do not prevent the exposure of other body parts which researchers are trying to avoid. A key characteristic of User Product Development method is a "systematic analysis of the relation between user, product, task, and environment," [11]. Once the designer accepts responsibility for the situation, the problem becomes the generator of the process. User needs and 


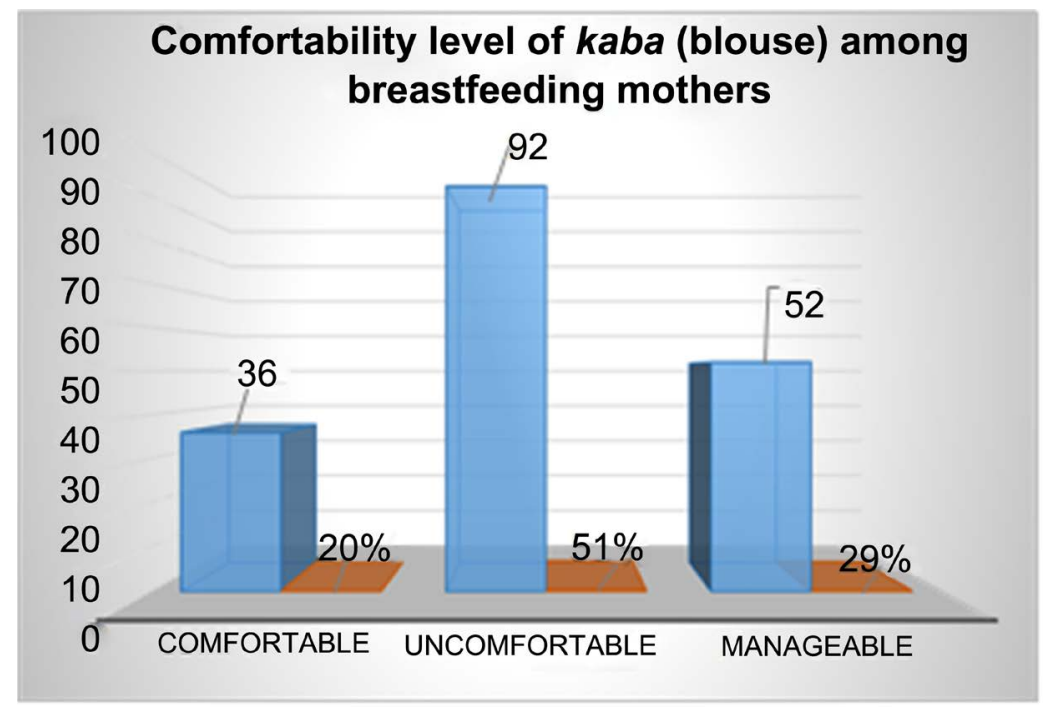

Figure 5. Bar chart representing comfortability level of the kaba (blouse) among breastfeeding mothers.

wants are analyzed, while the nature of the problem to be solved is being defined [8]. Specific functional properties with regards to ease of use of apparel is the ability of the apparel to be operated with one hand, allow for easy and quick access to the breast as well as versatility in the function of the garment [26].

\subsection{Preliminary Ideas}

In this creative phase of the project, a mood board (Figure 6) was created with pictures of the existing kaba designs. This made way for the adaptation and modification of design from existing ones.

\subsection{Design Refinement}

The kaba ensemble is one such apparel that can be sewn fitted through the incorporation of darts or made loose. Considering fit, mobility, comfort, protection and donning on or off as a functional purpose of an apparel by Lamb and Kallal, there is a need to consider the before, during and after use of the apparel so that the apparel is not exclusive to breastfeeding alone, but can be worn even after breastfeeding. Based on these, several kaba styles were sketched with ideas from the mothers as the product is supposed to be user centered. Openings, with the aid of fasteners such as zips and buttons were incorporated through the darts at the breast areas. These were made conspicuous and inconspicuous depending on the style line involved. Also, their placements were well scrutinized so as not to affect or hurt the baby in any way. The prototypes were developed from sketches 1, 2, 3, 5 and 6 of the preliminary sketches of Figure 7.

\subsection{Prototype Development}

The prototypes produced were chosen from existing kaba designs with the African print fabrics based on discussions with the mothers. Researchers' agree with 


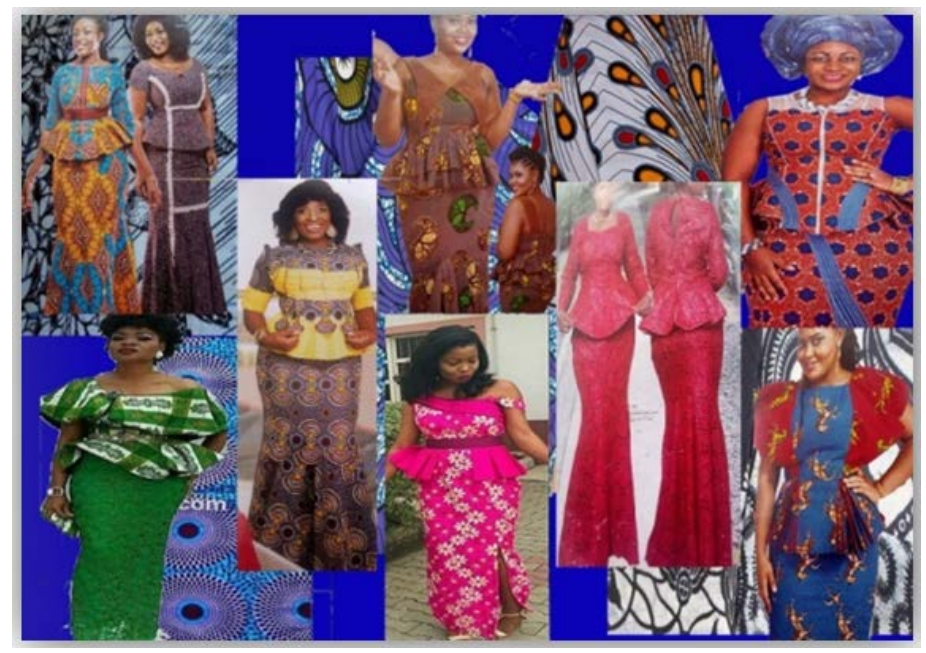

Figure 6. Moodboard of existing kaba designs.
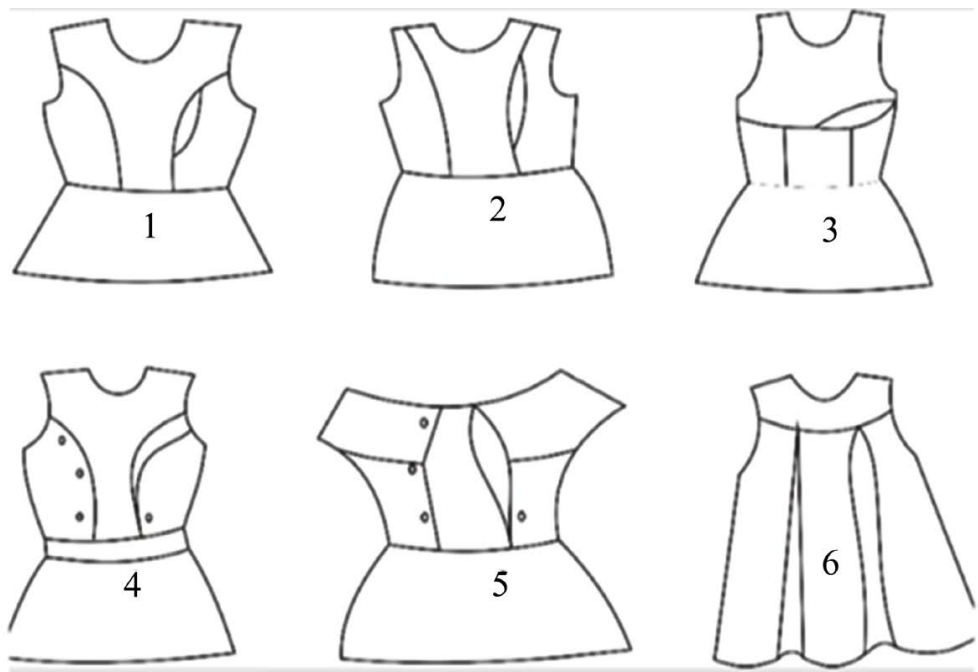

Figure 7. Preliminary sketches of zipper positions.

[25] that, necklines are an important feature of breastfeeding apparel in tops, dresses, tanks and bras. Scoop, V-neck and cross-over necklines are typical because they allow easy access for breastfeeding by simply pulling the garment down or up depending on the apparel feature. But the creation of openings at the breast areas was to give the mothers direct access to the breast without exposing other parts of their body's while keeping intact the necklines of their garments. Hence mothers can have the opportunity to have any neckline of their choice without thinking of how to breastfeed their infants in public. The fastenings that were used in designing the apparel were tested at this stage to ascertain their feasibility in their usage in the designed garment. Paper patterns of the five chosen styles were drafted using the users' measurements and later developed and given to the breastfeeding mothers for assessment. Below are the details of each design where (a) represents kaba in full style; and (b) represents openings created in the blouse (Figures 8-12). 


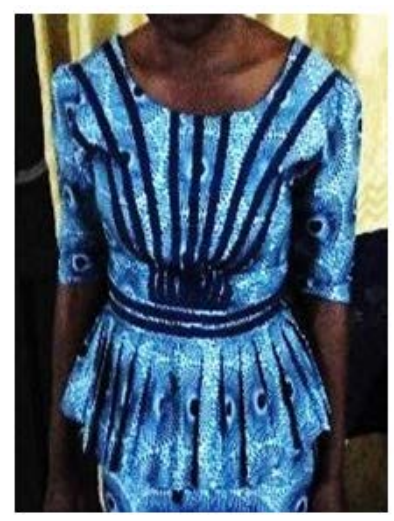

(a)

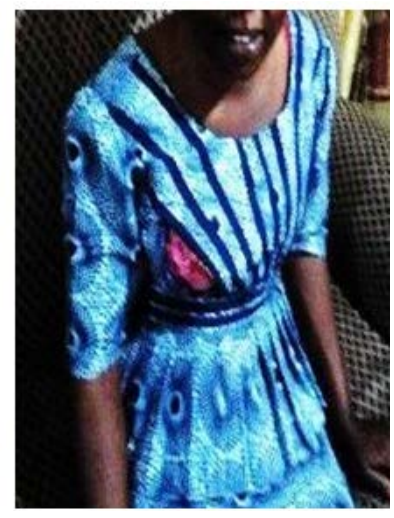

(b)

Figure 8. Design 1. 1) Design 1 is made up of a front and back round neckline; 2) Diagonal invisible zippers opening coming through the armhole dart at both breasts; 3) Bias stripes used as an illusion to the opening converging at the waist; 4) Knife pleated peplum attached at waist; 5) Set-in sleeve; 6) Centre back zipper opening.

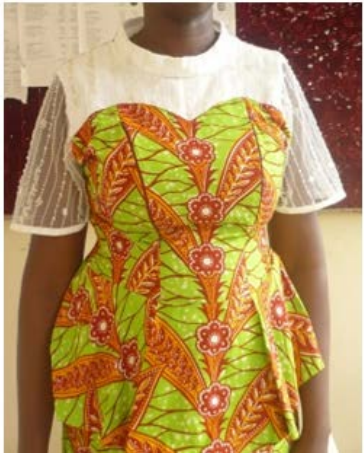

(a)

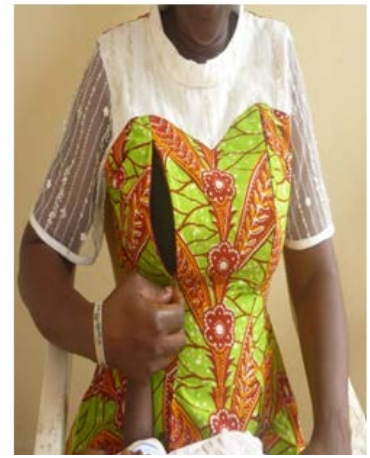

(b)

Figure 9. Design 2. 1) Design 2 is made of a high neckline mandarin collar styled; 2) Yoke made of lace; 3) Vertical invisible zipper opening in the princess line at both breasts; 4) Set-in sleeve made with contrasting colour lace fabric; 5) Shaped kaba; 6) Centre back invisible zipper opening.

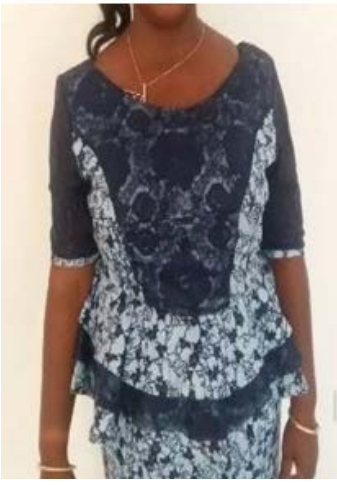

(a)

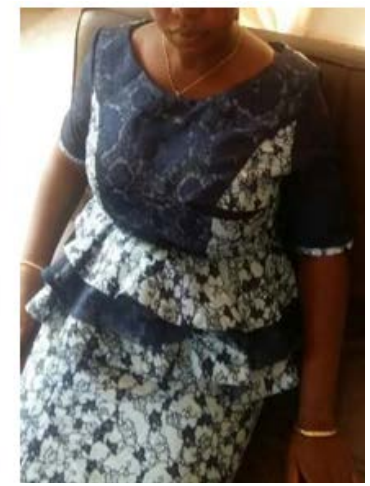

(b)

Figure 10. Design 3. 1) Design 3 is made up of a front and back round neckline; 2) Horizontal invisible zippers positioned in the armhole darts areas at both breasts; 3 ) Contrast lace fabric used as an illusion to the zipper opening; 4) Three tier knife pleated peplum attached at waist; 5) Set-in sleeve made in lace contrast fabric; 6) Centre back zipper opening. 


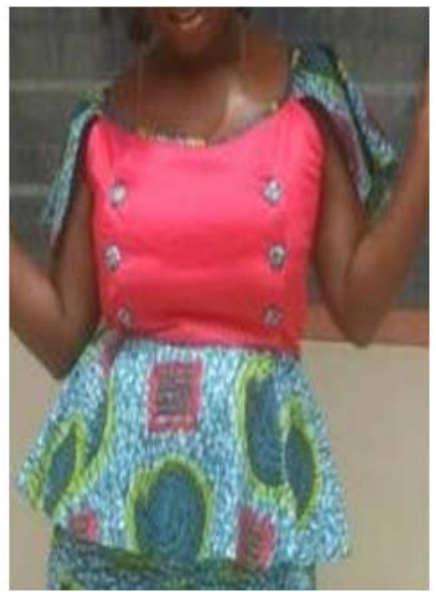

(a)

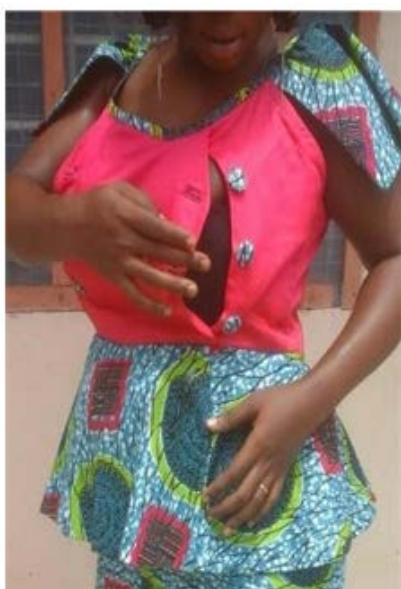

(b)

Figure 11. Design 4. 1) Design 4 is made of a raglan sleeve insert style; 2) Upper bodice made with contrasting plain cotton fabric; 3) Vertical opening fastened with covered buttons at both breasts; 4) Box pleated peplum attached at waist; 5) Centre back zipper opening.

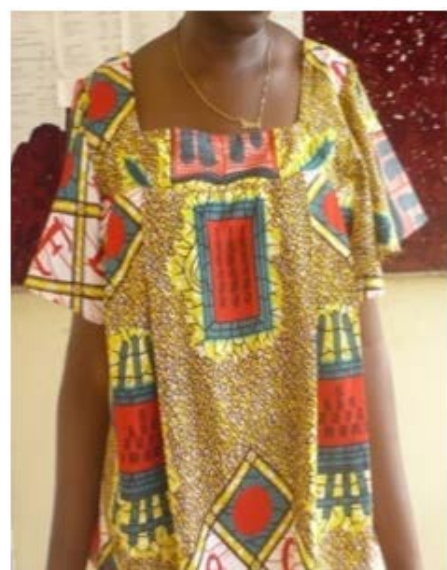

(a)

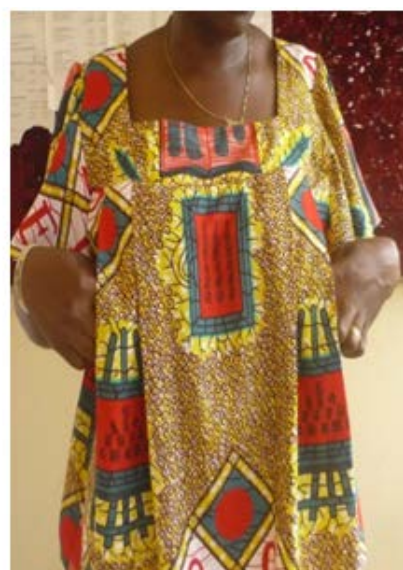

(b)

Figure 12. Design 5. 1) Design 5 is made of a front and back square yoke neckline; 2) Loose kaba blouse; 3) Pleated lower part attached at yoke; 4) Vertical opening underneath pleats at the breasts area; 5) Circular set-in sleeve style.

The five sets of redesigned kaba were given to three mothers to assess their suitability, comfortability and acceptability towards effective breastfeeding.

\section{Results and Discussion}

The fifth stage in Lamb and Kallal's apparel design framework (Figure 1) is the assessment of the prototypes according to the criteria of the problem identified. Thus, in ascertaining the suitability of the redesigned apparels for breastfeeding, it was found out that, the placement of zippers went a long way to help breastfeed confidently without exposing other body parts. Constructional features such as the use of darts, zipper openings in the horizontal, diagonal and vertical directions, button openings, sleeves, peplums, necklines and shape of the apparel 
were attributed to suitability in the use of the apparel.

User one applauded the fact that, the higher neckline of design two (Figure 9) suggested that there will be no barrier towards one's love for high necklines and breastfeeding. Though necklines are an important feature in lactating apparels as stipulated by [25] they can also be worn out of choice if the right approach is used in solving the stated problem. On the issue of breastfeeding being compromised by the apparel, all three users said breastfeeding was not compromised since it did not come with so much embellishment that could impede breastfeeding. The creation of openings through the darts was to maintain the shape of the wearer so as not to deviate from the fitting features of most of the Ghanaian $k a b a$ ensemble. For those mothers who preferred loose $k a b a$, as in design five (Figure 12), pleats were created to hide the openings. With this constructional feature, the users applauded the fact that it was easy to wear, very airy and user friendly. All the designs were seen to be very suitable for breastfeeding with much preference for the diagonal and vertical openings of design one (Figure 8) two (Figure 9) and four (Figure 11) respectively.

Furthermore, the ease of use of the apparel and the ease to fasten on and off easily without difficulty, led to the comfortable and confident breastfeeding of infants even in public places. Time commitment to breastfeed should enable mothers operate their apparels with one hand to allow for easy and quick access to the breast as well as versatile in the function of the garment [26]. According to the users, they were able to breastfeed their infants regardless of how the baby is held as the openings gave them the opportunity to open to the desired length to enable them bring out the breast to breastfeed. In as much as the comfortability of the mother is important, it must also be noted that infant's comfortability should also not be compromised in the choice of fasteners used as the choice of fastener could affect the comfortability of the baby. Hence, the size of buttons used for design four (Figure 11) could be much smaller to avoid infants' cheeks from resting on them. The designs according to the respondents were also very easy to fasten on and off such that it did not create any form of inconveniences to them. While all users felt that it was very easy using design five (Figure 12) as it had no fastener, user one and three felt that the use of zippers to produce design one (Figure 8), two (Figure 9) and three (Figure 10) were much easier to open and close. User two also thought otherwise with Design four (Figure 11) as she thinks it was much easier to use. User two also applauded the fact that there was no need for a third person to help unzip when it comes to breastfeeding either at home or in a public place. Therefore, in the comfortability of breastfeeding in the apparels, all five garments were said to be very comfortable with much preference for design one (Figure 8), two (Figure 9) and four (Figure 11).

The success of any designed product is the ability of it to be accepted by its intended users if they are to be implemented. The users felt that the idea of assisting breastfeeding mothers to breastfeed comfortably was great and are ever ready to accept its implementation. They saw the apparel as a novelty in designing kaba to assist breastfeeding mothers and are ready to embrace it. The moth- 
ers admitted the apparels were aesthetically pleasing though it may differ from person to person yet the intent of the apparel was duly understood. Most maternity and lactating apparels have limited longevity and thus could be used for a limited period of time after which the garment is rendered unserviceable when the baby is born or stops feeding [24]. The mothers liked the fact that the apparel could be used even after breastfeeding and said that unlike maternity wears that are abandoned after delivery, this design concept can even be used beyond breastfeeding since the openings were made invisible enough and the buttons, though serving a purpose, formed part of the design. They admitted that the process of replication can be made easily through knowledge in dart manipulation though it was their first time of hearing it.

\section{Conclusion}

Functional apparels are designed to help identified users feel comfortable in and around themselves. Hence, the functional breastfeeding kaba (blouse) apparels were successful for their intended purpose and that they were suitable, comfortable and aesthetically pleasing hence their acceptability to be implemented for use. This kaba idea has the potential of boosting the confidence of the Ghanaian woman towards breastfeeding without compromising on their expressive values. It can be concluded that respondents were satisfied in terms of design, construction and functionality as their confidence were boosted to breastfeed their babies in public places suggesting that maternity garments with functional features are very much vital.

\section{Recommendations}

Maternity garment manufacturing units or providers in each town/city should make use of this research outcome in order to satisfy breastfeeding mothers' comfortability, suitability and acceptability towards effective breastfeeding. The developed designs can also be used as a reference design concept by garment manufacturing units to bring variety in their delivery to these special group of consumers who have specific needs to enable them to fulfill their functional obligations during and after breastfeeding.

\section{Acknowledgements}

Authors are thankful to the breastfeeding mothers who were involved in the project and post-natal Hospital units for their co-operation during this study.

\section{Conflicts of Interest}

The authors declare no conflicts of interest regarding the publication of this paper.

\section{References}

[1] Condra, J. (2013) Encyclopedia of National Dress: Traditional Clothing around the 
World. ABC-CLIO, Santa Barbra.

[2] Khatri, K. and Srivastava, M. (2010) Development of Suitable Clothing for Lactating mothers and Assessment of Its Preference. Asian Journal of Home Science, 5, 221-225.

[3] Malik, T. (2012) Clothing Physiology and Comfort. Sakets Project Limited, Ahmedabad.

[4] Kaiser, S. (1990) The Social Psychology of Clothing: Symbolic Appearance in Context. 2nd Edition, Macmillan, New York.

[5] Gupta, D. (2011) Functional Clothing, Definition and Classification. Indian Journal of Fiber and Textile Research, 36, 321-326.

[6] Gupta, D. (2011) Design and Engineering of Functional Clothing. Indian Journal of Fiber and Textile Research, 36, 327-335.

[7] Curteza, A., Cretu, V., Macovei, L. and Poboronuic, M. (2014) Designing Functional Clothes for Persons with Locomotive Disabilities. Autex Research Journal, 14, 281-289.

[8] Lamb, J. and Kallal, M. (1992) A Conceptual Framework for Apparel Design. Clothing and Textile Research Journal, 10, 42-47. https://doi.org/10.1177\%2F0887302X9201000207

[9] Rosenbald-Wallin, E. (1985) User Oriented Product Development Applied to Functional Clothing Design. Applied Ergonomics, 16, 279-287. https://doi.org/10.1016/0003-6870(85)90092-4

[10] Suzana Barreto, M. (2012) Ergonomics, Design Universal and Fashion. Work, 41, 4733-4738. https://doi.org/10.3233/WOR-2012-1061-6059

[11] Kaulio, M.A. (1998) Customer, Consumer and User Involvement in Product Development: A Framework and a Review of Selected Methods. Total Quality Management, 9, 141-149. https://doi.org/10.1080/0954412989333

[12] May-Plumlee, T. and Pittman, A. (2002) Surgical Gown Requirements Capture: A Design Analysis Case Study. Journal of Textile and Apparel Technology and Management, 2, 1-10.

[13] Unruh, G.G. and Unruh, A. (1984) Curriculum Development: Problem, Process, and Progress. Mc Cutchan Publishing Corporation, Berkeley.

[14] Rouda, R. and Kusy M. (1995) Development of Human Resources. Needs Assessment-the First Step. Tappi Journal, 78, 255-257.

[15] Chae, M. and Schofield-Tomschin, S. (2010) Investigation of Design Characteristics and Regulatory Requirements for Snowboarding Helmets. International Journal of Fashion Design, Technology and Education, 3, 89-97. https://doi.org/10.1080/17543266.2010.493530

[16] Bye, E. and Hakala, L. (2005) Sailing Apparel for Women: A Design Development Case Study. Clothing and Textile Research Journal, 23, 45-55. https://doi.org/10.1177\%2F0887302X0502300104

[17] Dickson, M.A. and Pollack, A. (2000) Clothing and Identity among Female In-Line Skaters. Clothing and Textiles Research Journal, 18, 65-72. https://doi.org/10.1177\%2F0887302X0001800201

[18] Dzramedo, E.B. (2009) Clothing and Fashion in Ghanaian Culture: A Case Study among the Akans. Unpublished Dissertation, University of Science and Technology (KNUST), Kumasi-Ghana.

[19] Spradely, J.P. (1972) Culture and Cognition: Rules, Maps and Plans. Chandler Publishing, San Francisco. 
[20] Blumer, H. (1969) Symbolic Interactionism: Perspective and Methods. Prentice Hall, Eaglewood Cliff.

[21] Howard, E.S. Sarpong, G.D. and Amankwa, A.M. (2012) Symbolic Significance of African Prints: A Dying Phenomenon in Contemporary Print Designs in Ghana. International Journal of Innovative Research and Development, 1, 609-624.

[22] Gott, S. and Loughran, K. (2010) Contemporary African Fashion. Indiana University Press, Bloomington.

[23] Gott, S. (2009) Asante Hightimers and the Fashionable Display of Women's Wealth in Contemporary Ghana. Journal of Dress and Culture, 13, 141-176. https://doi.org/10.2752/175174109X414259

[24] Singh, A. (2012) Designing of Maternity and Nursing Apparels for Expectant and Lactating Mothers. Chhatrapati Sahuji Maharaj University, Kalyanpur. http://hdl.handle.net/10603/268489

[25] Choi, H., Choi, J., and Kim, S. (2000) A Study of the Development of Nursing Brassiers. Journal of Korean Society of Clothing and Textiles, 24, 918-927.

[26] Gordon, L.A. (2015) The Development of Design Requirements for Breastfeeding Apparel: A User Oriented Product Development Approach. Master's Theses, University of Minnesota Digital Conservancy, Minneapolis and Saint Paul. 